\title{
Parallel multilevel solvers for the cardiac electro-mechanical coupling
}

\author{
P. Colli Franzone ${ }^{\mathrm{a}}$, L. F. Pavarino ${ }^{\mathrm{b}}$, S. Scacchi ${ }^{\mathrm{b}}$ \\ ${ }^{a}$ Dipartimento di Matematica, Università di Pavia, Via Ferrata, 27100 Pavia, Italy. E-mail: \\ colli@imati.cnr.it \\ ${ }^{b}$ Dipartimento di Matematica, Università di Milano, Via Saldini 50, 20133 Milano, Italy. E-mail: \\ luca.pavarino@unimi.it, simone.scacchi@unimi.it.
}

\begin{abstract}
We develop a parallel solver for the cardiac electro-mechanical coupling. The electric model consists of two non-linear parabolic partial differential equations (PDEs), the so-called Bidomain model, which describes the spread of the electric impulse in the heart muscle. The two PDEs are coupled with a non-linear elastic model, where the myocardium is considered as a nearly-incompressible transversely isotropic hyperelastic material. The discretization of the whole electro-mechanical model is performed by Q1 finite elements in space and a semiimplicit finite difference scheme in time. This approximation strategy yields at each time step the solution of a large scale ill-conditioned linear system deriving from the discretization of the Bidomain model and a non-linear system deriving from the discretization of the finite elasticity model. The parallel solver developed consists of solving the linear system with the Conjugate Gradient method, preconditioned by a Multilevel Schwarz preconditioner, and the non-linear system with a Newton-Krylov-Algebraic Multigrid solver. Three-dimensional parallel numerical tests on a Linux cluster show that the parallel solver proposed is scalable and robust with respect to the domain deformations induced by the cardiac contraction.
\end{abstract}

Keywords: Cardiac electromechanics, Bidomain model, Finite Elasticity, Multilevel Schwarz preconditioners, Algebraic Multigrid

\section{Introduction}

We develop a parallel solver based on algebraic multigrid and multilevel Schwarz methods for the solution of the cardiac electro-mechanical coupling model. This model consists of the Bidomain equations (electrical model), a degenerate system of parabolic partial differential equations modeling the cardiac bioelectrical activity, coupled with a quasi-static mechanical model, describing the contraction and relaxation of the cardiac muscle during a heart beat.

The numerical approximation of the cardiac electro-mechanical coupling is a challenging multiphysics problem, because the space and time scales associated with the electrical and mechanical models are very different. The discretization of the model by finite elements in space and semi-implicit finite difference splitting methods in time yields at each time 
step the solution of a large ill-conditioned linear system, deriving from the discretization of the Bidomain equations, and of a non-linear system, deriving from the discretization of the non-linear elasticity equations.

Many studies have been devoted to the development of efficient solvers and preconditioners for the Bidomain model, see e.g. $[9,14,30,32,44,31,40,54,42,43,58,62,63]$ and the surveys $[37,60]$, but the robustness of these methods with respect to the domain deformation induced by the mechanical feedback has not been demonstrated yet. In the last years, several works have been devoted to the development of models for the mechanical cardiac contraction and to the numerical simulations of the electro-mechanical coupling models, see e.g. $[22,25,12,23,36,38,49,34,55]$. A few studies have focused on the development of efficient solvers for the quasi-static mechanical model, see [36, 59] for a parallel GMRES solver and [24, 25, 23] for parallel direct solvers. The majority of the simulation studies of the cardiac mechanics, have used very coarse mechanical meshes in comparison with the standard electrical meshes. Thus, the solution of the quasi-static nonlinear mechanical model is performed solving the linear Jacobian system by direct methods, at each Newton iteration. However, in the recent paper [38], it has been shown that, when complex active tension development model are used, stability issues may arise in case of too coarse mechanical meshes, and fine meshes are needed also for the mechanical model.

The first aim of the present paper is to study the scalability and robustness, with respect to mechanically induced domain deformations, of Multilevel Schwarz methods for the Bidomain system. The second aim is to study the scalability of Algebraic Multigrid preconditioners for the linear Jacobian system arising at each Newton iteration during the solution of the non-linear elasticity equations in the mechanical model.

\section{Mathematical models}

\subsection{Mechanical model}

Let us denote the material coordinates of the undeformed or reference cardiac domain by $\mathbf{X}=\left(X_{1}, X_{2}, X_{3}\right)^{T}$, the spatial coordinates of the deformed cardiac domain by $\mathbf{x}=$ $\left(x_{1}, x_{2}, x_{3}\right)^{T}$ and the region occupied by the undeformed and deformed, at time $t$, cardiac domains by $\hat{\Omega}$ and $\Omega(t)$, respectively. We denote by Div and div (Grad and grad) the material and spatial divergence (gradient) of a vector (scalar), respectively. From a mechanical point of view, the cardiac tissue is modeled as a non-linear elastic material. The deformation gradient tensor $\mathbf{F}$ and its determinant are given by

$$
\mathbf{F}(\mathbf{X}, t)=\left\{F_{i j}\right\}=\left\{\frac{\partial x_{i}}{\partial X_{j}} \quad i, j=1,2,3\right\}, \quad J(\mathbf{X}, t)=\operatorname{det} \mathbf{F}(\mathbf{X}, t)
$$

The Cauchy-Green deformation tensor $\mathbf{C}$ and Lagrange-Green strain tensor $\mathbf{E}$ are

$$
\mathbf{C}=\mathbf{F}^{T} \mathbf{F} \quad \text { and } \quad \mathbf{E}=\frac{1}{2}(\mathbf{C}-\mathbf{I})
$$

where I denotes the identity matrix. 
We first assume that the time-dependent inertial term in the governing elastic wave equation may be neglected, see e.g. [25, 26, 27, 33, 61, 6]. Thus, the quasi-static Cauchy's equation of equilibrium, without body force, in term of the Cauchy stress tensor $\sigma$ is given by div $\sigma=\mathbf{0}$, in $\Omega(t)$ and in the coordinates of the deformed body satisfy the steady-state force equilibrium equation

$$
\operatorname{Div}(\mathbf{F S})=\mathbf{0}, \quad \mathbf{X} \in \hat{\Omega},
$$

where $\mathbf{S}=\left\{s_{i j}\right\}=J \mathbf{F}^{-1} \sigma \mathbf{F}^{-T}$ is the second Piola-Kirchoff stress tensor. The tensor $\mathbf{S}$ is given by the sum of a passive elastic component $\mathbf{S}^{\text {pas }}$ and a biochemically generated active component $\mathbf{S}^{a c t}$, i.e. $\mathbf{S}=\mathbf{S}^{\text {pas }}+\mathbf{S}^{a c t}$, as done in many previous studies, see e.g. $[21,59,27]$. An alternative multiplicative strategy for combining the passive $\mathbf{S}^{\text {pas }}$ and active $\mathbf{S}^{\text {act }}$ components has been recently proposed in [7], see also [1, 36, 48].

The passive component $\mathbf{S}^{\text {pas }}$ is computed from a suitable strain energy function $W$ and the Green Lagrange strain $\mathbf{E}$

$$
S_{i j}^{p a s}=\frac{1}{2}\left(\frac{\partial W}{\partial E_{i j}}+\frac{\partial W}{\partial E_{j i}}\right) \quad i, j=1,2,3 .
$$

A wide variety of strain energy functions $W$ have been proposed and adopted in the literature, see e.g. $[11,13,15,18,33,45,49,52,57]$. We recall that the cardiac tissue consists of an arrangement of fibers that rotate counterclockwise from epi- to endocardium, and that have a laminar organization modeled as a set of muscle sheets running radially from epito endocardium, e.g. [28, 57]. In this paper, we choose to model the myocardium as a transversely isotropic hyperelastic material, with the exponential strain energy function [59]

$$
\begin{gathered}
W=\frac{1}{2} c\left(e^{Q}-1\right), \\
Q=b_{l l} E_{l l}^{2}+b_{t n}\left(E_{n n}^{2}+E_{t t}^{2}+2 E_{n t}^{2}\right)+2 b_{l t}\left(E_{l t}^{2}+E_{l n}^{2}\right),
\end{gathered}
$$

where the Lagrange-Green strain tensor is referred to the orthogonal local fiber coordinate system, consisting of the fiber direction $(l)$, and two others orthogonal cross fiber directions. The material constant $c$ scales the stress, $b_{l l}$ and $b_{t n}$ scale the material stiffness in the fiber and the two cross fiber directions, and $b_{l t}$ scales the material rigidity under shear in the fiber-transverse plane.

The myocardium is modelled as nearly-incompressible material and, following [59], we add a bulk modulus $K$ multiplying a volume change penalization term into the strain energy

$$
W=\frac{1}{2} c\left(e^{Q}-1\right)+K(\sqrt{\operatorname{det}(C)}-1)^{2} .
$$

We close the quasi-static mechanical model (1) by imposing a prescribed displacement on a Dirichlet boundary $\mathbf{x}(\mathbf{X})=\hat{\mathbf{x}}(\mathbf{X}), \mathbf{X} \in \partial \hat{\Omega}_{D}$ and no traction force on a Neumann boundary $\mathbf{F S} \mathbf{N}=\mathbf{0}, \mathbf{X} \in \partial \hat{\Omega}_{N}$.

The contraction of the ventricles results from the active tension generated by the model of myofilements dynamics activated by calcium (see e.g. [21, 46, 35, 47] for electro-contraction 
coupling models). We assume that the generated active force acts only in the direction of the fiber, as in the other works $[38,61,13]$, hence, according to [20, Ch. 10], the active Cauchy stress is expressed as

$$
\sigma^{a c t}=T_{a} \mathbf{a}_{l} \otimes \mathbf{a}_{l},
$$

where $\mathbf{a}_{l}$ is a unit vector parallel to the local fiber direction and $T_{a}$ is the active stress related to the deformed domain. Let us denote by $\hat{\mathbf{a}}_{l}(\mathbf{X})$ and $\hat{\mathbf{a}}_{t}(\mathbf{X})$ the unit vectors parallel and across the local fiber direction in the reference configuration. Then, $\mathbf{a}_{l}=\mathbf{F} \hat{\mathbf{a}}_{l} /\left\|\mathbf{F} \hat{\mathbf{a}}_{l}\right\|$ is the unit vector parallel to the local fiber in the deformed configuration and it holds $\mathbf{a}_{l}=\mathbf{F} \hat{\mathbf{a}}_{l} / \sqrt{\hat{\mathbf{a}}_{l}^{T} \mathbf{C} \hat{\mathbf{a}}_{l}^{T}}$. In terms of the principal axes of the reference configuration, we obtain:

$$
\mathbf{a}_{l} \otimes \mathbf{a}_{l}=\frac{\mathbf{F} \hat{\mathbf{a}}_{l} \otimes \mathbf{F} \hat{\mathbf{a}}_{l}}{\left\|\mathbf{F} \hat{\mathbf{a}}_{l}\right\|^{2}}=\frac{\mathbf{F} \hat{\mathbf{a}}_{l} \hat{\mathbf{a}}_{l}^{T} \mathbf{F}^{T}}{\hat{\mathbf{a}}_{l}^{T} \mathbf{C} \hat{\mathbf{a}}_{l}} .
$$

Then, the corresponding second Piola-Kirchhoff active stress component is given by

$$
\mathbf{S}^{a c t}=J \mathbf{F}^{-1} \sigma^{a c t} \mathbf{F}^{-T}=J T_{a} \frac{\hat{\mathbf{a}_{l}} \otimes \hat{\mathbf{a}_{l}}}{\hat{\mathbf{a}}_{l}^{T} \mathbf{C} \hat{\mathbf{a}}_{l}} .
$$

In this simulation study, we assume that the biochemically generated active tension $T_{a}$ response is modelled by the following simple dynamics proposed by Nash and Panfilov in $[34]$

$$
\frac{\partial T_{a}}{\partial t}=Q\left(V, T_{a}\right)=\epsilon(V)\left(k_{T_{a}} V-T_{a}\right), \text { with } \epsilon(V)= \begin{cases}1 & \text { for } V<0.05 \\ 10 & \text { for } V \geq 0.05\end{cases}
$$

where $k_{T_{a}}=47.9 \mathrm{kPa}$ controls the amplitude of the active stress twitch and the function $\epsilon(V)$ controls the delay in the development $(V<0.05)$ and recovery $V \geq 0.05$ of active stress with respect to the normalized action potential $V$.

\subsection{Bidomain model}

The macroscopic Bidomain representation of the cardiac tissue volume $\Omega$ is obtained by considering the superposition of two anisotropic continuous media, the intra- (i) and extra(e) cellular media, coexisting at every point of the tissue and separated by a distributed continuous cellular membrane; see e.g. $[10,41]$ for a derivation of the Bidomain model from homogenization of an assembling of cellular models.

The anisotropy of the intra- and extracellular media, related to the macroscopic arrangement of the cardiac myocytes in the fiber structure, is described by the anisotropic conductivity tensors $D_{i}(\mathbf{x})$ and $D_{e}(\mathbf{x})$, respectively, defined in (8) below.

In order to take into account the mechanical deformation of the tissue, following [34, $38,61]$ we will now introduce the parabolic-parabolic formulation of the Bidomain system on the reference cardiac domain $\hat{\Omega}$. Given an applied extracellular current per unit volume $I_{\text {app }}^{e}: \hat{\Omega} \times(0, T) \rightarrow \mathbb{R}$, and initial conditions $v_{0}: \hat{\Omega} \rightarrow \mathbb{R}, w_{0}: \hat{\Omega} \rightarrow \mathbb{R}^{N_{w}}$, find the intra- and extracellular potentials $u_{i, e}: \hat{\Omega} \times(0, T) \rightarrow \mathbb{R}$, the transmembrane potential $v=u_{i}-u_{e}$ : 
$\hat{\Omega} \times(0, T) \rightarrow \mathbb{R}$, the gating and ionic concentrations variables $(w, c): \hat{\Omega} \times(0, T) \rightarrow \mathbb{R}^{N_{w}} \times \mathbb{R}^{N_{c}}$ such that

$$
\begin{cases}c_{m} \frac{\partial v}{\partial t}-J^{-1} \operatorname{Div}\left(J \mathbf{F}^{-1} D_{i} \mathbf{F}^{-T} \operatorname{Grad} u_{i}\right)+I_{i o n}(v, w, c)=0 & \text { in } \hat{\Omega} \times(0, T) \\ -c_{m} \frac{\partial v}{\partial t}-J^{-1} \operatorname{Div}\left(J \mathbf{F}^{-1} D_{e} \mathbf{F}^{-T} \operatorname{Grad} u_{i}\right)-I_{i o n}(v, w, c)=I_{a p p}^{e} & \text { in } \hat{\Omega} \times(0, T) \\ \frac{\partial w}{\partial t}-R(v, w)=0, \quad \frac{\partial c}{\partial t}-S(v, w, c)=0, & \text { in } \hat{\Omega} \times(0, T) \\ \hat{\mathbf{n}}^{T} \mathbf{F}^{-1} D_{i, e} \mathbf{F}^{-T} \operatorname{Grad} u_{i, e}=0 & \text { in } \partial \hat{\Omega} \times(0, T) \\ v(\mathbf{x}, 0)=v_{0}(\mathbf{x}), \quad w(\mathbf{x}, 0)=w_{0}(\mathbf{x}), \quad c(\mathbf{x}, 0)=c_{0}(\mathbf{x}) & \text { in } \hat{\Omega},\end{cases}
$$

where $\mathbf{F}$ is the deformation gradient tensor and we have assumed an insulated cardiac boundary $\partial \hat{\Omega}$. For an applied extracellular current $I_{a p p}^{e}$ satisfying the compatibility condition $\int_{\Omega} I_{a p p}^{e} \mathrm{dx}=0$, this system uniquely determines $v$, while the potentials $u_{i}$ and $u_{e}$ are defined only up to the same additive time-dependent constant related to the reference potential. We choose this potential to be the average extracellular potential in the cardiac volume by imposing $\int_{\Omega} u_{e} \mathrm{dx}=0$. The nonlinear reaction term $I_{i o n}$ and the ODE system for the gating variables $w$ are given by the ionic membrane model; here we will consider the Luo-Rudy I (LR1) membrane model [29].

The conductivity tensors $D_{i}(\mathbf{x})$ and $D_{e}(\mathbf{x})$ at any point $\mathbf{x} \in \Omega$ are defined as

$$
D_{i, e}(\mathbf{x})=\sigma_{l}^{i, e} \mathbf{a}_{l}(\mathbf{x}) \mathbf{a}_{l}^{T}(\mathbf{x})+\sigma_{t}^{i, e} \mathbf{a}_{t}(\mathbf{x}) \mathbf{a}_{t}^{T}(\mathbf{x})+\sigma_{n}^{i, e} \mathbf{a}_{n}(\mathbf{x}) \mathbf{a}_{n}^{T}(\mathbf{x})
$$

Here $\mathbf{a}_{l}(\mathbf{x}), \mathbf{a}_{t}(\mathbf{x}), \mathbf{a}_{n}(\mathbf{x})$, is a triplet of orthonormal principal axes with $\mathbf{a}_{l}(\mathbf{x})$ parallel to the local fiber direction, $\mathbf{a}_{t}(\mathbf{x})$ and $\mathbf{a}_{n}(\mathbf{x})$ tangent and orthogonal to the radial laminae, respectively, and both being transversal to the fiber axis (see e.g. LeGrice et al. [28]). Moreover, $\sigma_{l}^{i, e}, \sigma_{t}^{i, e}, \sigma_{n}^{i, e}$ are the conductivity coefficients in the intra- and extracellular media measured along the corresponding directions $\mathbf{a}_{l}, \mathbf{a}_{t}, \mathbf{a}_{n}$. In this work, the electric conduction of the cardiac tissue is model as an axi-symmetric anisotropic media with respect to the local finer direction, i.e. $\sigma_{n}^{i, e}=\sigma_{t}^{i, e}$. Hence, using the orthogonality of the principal axes the conductivity tensors can be written as

$$
D_{i, e}(\mathbf{x})=\sigma_{t}^{i, e} \mathbf{I}+\left(\sigma_{l}^{i, e}-\sigma_{t}^{i, e}\right) \mathbf{a}_{l}(\mathbf{x}) \mathbf{a}_{l}^{T}(\mathbf{x}) .
$$

The computation of the tensors $\mathbf{F}^{-1}(\mathbf{X}) D_{i, e}(\mathbf{x}) \mathbf{F}^{-T}(\mathbf{X})$ must be performed on the reference configuration $\hat{\Omega}$. Denoting by $\hat{\mathbf{a}}_{l}(\mathbf{X})$ the unit vector parallel to the local fiber direction in the reference configuration, then we recall that, from (5), it holds $\mathbf{a}_{l} \mathbf{a}_{l}^{T}=\frac{\mathbf{F} \hat{\mathbf{a}}_{l} \hat{\mathbf{a}}_{l}^{T} \mathbf{F}^{T}}{\hat{\mathbf{a}}_{l}^{T} \mathbf{C} \hat{\mathbf{a}}_{l}}$. Considering the product $\mathbf{F}^{-1}(\mathbf{X}) D_{i, e}(\mathbf{x}) \mathbf{F}^{-T}(\mathbf{X})$ and assuming that the conductivity coefficients refer to the deformed configuration, we finally obtain

$$
\left(\mathbf{F}^{-1} D_{i, e} \mathbf{F}^{-T}\right)(\mathbf{X})=\sigma_{t}^{i, e} \mathbf{C}^{-1}(\mathbf{X})+\left(\sigma_{l}^{i, e}-\sigma_{t}^{i, e}\right) \frac{\hat{\mathbf{a}}_{l}(\mathbf{X}) \hat{\mathbf{a}}_{l}^{T}(\mathbf{X})}{\hat{\mathbf{a}}_{l}^{T}(\mathbf{X}) \mathbf{C}(\mathbf{X}) \hat{\mathbf{a}}_{l}(\mathbf{X})}
$$


Instead, if the conductivity coefficients refer to the reference configuration, than the influence of the deformation is taken into account as

$$
\left(\mathbf{F}^{-1} D_{i, e} \mathbf{F}^{-T}\right)(\mathbf{X})=\sigma_{t}^{i, e} \mathbf{C}^{-1}(\mathbf{X})+\left(\sigma_{l}^{i, e}-\sigma_{t}^{i, e}\right) \mathbf{F}^{-1} \hat{\mathbf{a}}_{l}(\mathbf{X}) \hat{\mathbf{a}}_{l}^{T}(\mathbf{X}) \mathbf{F}^{-T}
$$

\subsection{Coupled electro-mechanical model}

In summary, our coupled electro-mechanical model consists of the mechanical contraction model (1), (2), (6), coupled through the tensors $\mathbf{F}$ and $J$ with the Bidomain model (7), (11), written on the reference configuration.

The well-posedness of the coupling of the Bidomain model and the mechanical contraction model based on the active stress approach is still an open problem, as well as the convergence of its finite element approximation. A first investigation of the strong ellipticity condition of the passive and active strain energy function can be found in [39] and [2]; see also [18]. A partial result on the solvability of the coupled problem has been established recently in [3] for passive linear strain energy function and for contraction models based on the active strain approach.

\section{Discretization and numerical methods}

The coupled electro-mechanical model is then discretized in time and space as follows. The time discretization is performed by a semi-implicit splitting method. At each time step,

1- given $v^{n}, w^{n}, c^{n}, T_{a}^{n}$, solve the ODEs of the membrane model and active force equation with a first order IMEX method to compute the new $w^{n+1}, c^{n+1}, T_{a}^{n+1}$

$$
\begin{aligned}
& w^{n+1}=w^{n}+\Delta t R\left(v^{n}, w^{n+1}\right) \\
& c^{n+1}=c^{n}+\Delta t S\left(v^{n}, w^{n+1}, c^{n}\right) \\
& T_{a}^{n+1}=T_{a}^{n}+\Delta t Q_{T}\left(v^{n}, T_{a}^{n+1}\right),
\end{aligned}
$$

2- given $T_{a}^{n+1}$ solve the mechanical problem to compute the new deformed coordinates $\mathbf{x}^{n+1}$, providing the new deformation gradient tensor $\mathbf{F}_{n+1}$

$$
\operatorname{Div}\left(\mathbf{F}_{n+1} \mathbf{S}_{n+1}\right)=0
$$

3- given $w^{n+1}, c^{n+1}, \mathbf{F}_{n+1}$, and $J_{n+1}=\operatorname{det}\left(\mathbf{F}_{n+1}\right)$ solve the Bidomain system with a first order IMEX method computing the new electric potentials $u_{i}^{n+1}, u_{e}^{n+1}, v^{n+1}=u_{i}^{n+1}-$ $u_{e}^{n+1}$

$$
\begin{aligned}
c_{m} \frac{v^{n+1}}{\Delta t}-J_{n+1}^{-1} \operatorname{Div}\left(J_{n+1} \mathbf{F}_{n+1}^{-1} D_{i} \mathbf{F}_{n+1}^{-T} \operatorname{Grad} u_{i}^{n+1}\right) & =c_{m} \frac{v^{n}}{\Delta t}-I_{i o n}\left(v^{n}, w^{n+1}, c^{n+1}\right) \\
-c_{m} \frac{v^{n+1}}{\Delta t}-J_{n+1}^{-1} \operatorname{Div}\left(J_{n+1} \mathbf{F}_{n+1}^{-1} D_{e} \mathbf{F}_{n+1}^{-T} \operatorname{Grad} u_{e}^{n+1}\right) & =-c_{m} \frac{v^{n}}{\Delta t}+I_{\text {ion }}\left(v^{n}, w^{n+1}, c^{n+1}\right)+I_{a p p}^{e, n+1}
\end{aligned}
$$


For the space discretization, we use structured grids of hexahedral isoparametric $Q_{1}$ finite elements in space, for both the non-linear elasticity system and the Bidomain equations. In this work, we consider a bulk modulus of $K=200 k P a$, so that the non-linear elasticity system is only moderately almost-incompressible and our pure displacement formulation is still appropriate. For larger values of $K$, the elasticity system becomes increasingly almostincompressible and volumetric locking affects both the accuracy of the numerical solution and the robustness of our iterative solver (described below). In such cases, we should adopt a mixed formulation with both displacements and pressures (see [5]) or a B-bar method (see [19]) or a splitting of the deformation into volumetric and isochoric parts (see [17]).

Due to the employed discretization strategy, at each time step, the main computational effort consists of

1- solving the non-linear system deriving from the discretization of the mechanical problem. We use the Newton method, with GMRES for the linear Jacobian system, preconditioned by an Algebraic Multigrid preconditioner [16].

2- solving the linear system deriving from the discretization of the Bidomain model. We use the Conjugate Gradient (CG) method preconditioned by the Block Jacobi (BJ) or the Multilevel Hybrid Schwarz preconditioner with L levels (MHS(L)), studied in $[50,51]$. See also $[53,56]$ for a general introduction to these methods. Inexact ILU(0) local solvers are used for the local problems on the subdomains.

\section{Numerical results}

In this section, we present the results of parallel numerical experiments performed on the Linux Cluster IBM SP6/5376 of the Cineca Consortium (www.cineca.it). Our FORTRAN-90 code is based on the parallel library PETSc [4], from the Argonne National Laboratory.

Domain geometry and fiber structure. The domain $\hat{\Omega}=\Omega(0)$ is the image of a cartesian slab using ellipsoidal coordinates, yielding a portion of truncated ellipsoid. The family of truncated ellipsoids is described by the parametric equations

$$
\begin{cases}x=a(r) \cos \theta \cos \phi & \phi_{\min } \leq \phi \leq \phi_{\max } \\ y=b(r) \cos \theta \sin \phi & \theta_{\min } \leq \theta \leq \theta_{\max } \\ z=c(r) \sin \theta & 0 \leq r \leq 1\end{cases}
$$

where $a(r)=a_{1}+r\left(a_{2}-a_{1}\right), b(r)=b_{1}+r\left(b_{2}-b_{1}\right), c(r)=c_{1}+r\left(c_{2}-c_{1}\right)$, and $a_{i}, b_{i}, c_{i}, i=$ 1,2 are given coefficients determining the main axes of the ellipsoid. The fibers rotate intramurally linearly with the depth for a total amount of $120^{\circ}$ proceeding counterclockwise from epicardium to endocardium. More precisely, in a local ellipsoidal reference system $\left(\mathbf{e}_{\phi}, \mathbf{e}_{\theta}, \mathbf{e}_{r}\right)$, the fiber direction $\mathbf{a}_{l}(\mathbf{x})$ at a point $\mathbf{x}$ is given by

$$
\mathbf{a}_{l}(\mathbf{x})=\mathbf{e}_{\phi} \cos \alpha(r)+\mathbf{e}_{\theta} \sin \alpha(r), \quad \text { with } \quad \alpha(r)=\frac{2}{3} \pi(1-r)-\frac{\pi}{4}, \quad 0 \leq r \leq 1 .
$$




\begin{tabular}{r|r|rrr}
\multicolumn{5}{c}{ Mechanical solver - AMG preconditioner } \\
\hline procs & dof & nit & lit & time \\
\hline 8 & 107,811 & 2 & 42 & 12.06 \\
27 & 352,947 & 2 & 42 & 16.70 \\
64 & 823,875 & 2 & 39 & 23.45 \\
125 & $1,594,323$ & 2 & 39 & 30.66 \\
216 & $2,738,019$ & 2 & 40 & 49.12 \\
343 & $4,328,691$ & 2 & 39 & 92.93 \\
512 & $6,440,067$ & 2 & 40 & 75.09 \\
\hline
\end{tabular}

Table 1: Weak scaling test, Mechanical solver with AMG preconditioner. Number of processors (procs), Degrees of freedom (dof), average Newton iterations (nit) per time step, average GMRES iteration counts (lit) per Newton iteration and time step, average CPU time in seconds (time) per time step.

Conductivity coefficients and strain energy function parameters. In order to reduce the parameter calibration, we assume that the cardiac tissue electric and mechanic behavior is transversely isotropic or axisymmetric with respect to the local fiber direction.

The values of the conductivity coefficients used in all the numerical tests are the following:

$$
\begin{array}{ll}
\sigma_{l}^{i}=3 . m \Omega^{-1} \mathrm{~cm}^{-1} & \sigma_{l}^{e}=2 . m \Omega^{-1} \mathrm{~cm}^{-1} \\
\sigma_{t}^{i}=\sigma_{n}^{i}=0.31525 m \Omega^{-1} \mathrm{~cm}^{-1} & \sigma_{t}^{e}=\sigma_{n}^{e}=1.3514 \mathrm{~m}^{-1} \mathrm{~cm}^{-1} .
\end{array}
$$

This choice of parameters yields physiological propagation velocities of the excitation wavefront along and across fiber of about of 0.05 and $0.03 \mathrm{~cm} \mathrm{~ms}^{-1}$, see e.g. [8]. In the transversely isotropic strain energy function (3) the values of the parameters are chosen as in the original work [59], i.e. $c=1.76 \mathrm{kPa}, b_{l l}=18.5, b_{t t}=b_{n n}=b_{t n}=b_{n t}=3.58, b_{l t}=b_{l n}=1.63$ and $K=200 k P a$.

Mesh hierarchy. We denote the cartesian mesh used to discretize our domains by $\mathcal{T}=\mathcal{T}_{i} \cdot \mathcal{T}_{j} \cdot \mathcal{T}_{k}$, indicating the number of elements in each coordinate direction. This notation applies to both fine and coarse meshes. When we scale up the mesh by a factor $c$, for brevity we define $c \mathcal{T}=c \mathcal{T}_{i} \cdot c \mathcal{T}_{j} \cdot c \mathcal{T}_{k}$, i.e. the number of elements in $c \mathcal{T}$ is $c^{3}$ times the number of elements in $\mathcal{T}$.

Stimulation site, initial and boundary conditions. The depolarization process is started by applying a cathodal extracellular stimulus of $I_{a p p}^{e}=-200 \mathrm{~mA} / \mathrm{cm}^{3}$ lasting $1 \mathrm{~ms}$ on a small volume of $0.4 \times 0.4 \times 0.2 \mathrm{~mm}^{3}$ located in the center of the endocardial surface. The initial conditions are at resting values for all the potentials and LR1 gating variables, while the boundary conditions are for insulated tissue. In all simulations, the fine mesh size is $h=0.01 \mathrm{~cm}$. The time step size is $\Delta t=0.05 \mathrm{~ms}$.

\subsection{Test 1: weak scaling}

We first consider a weak scaling test on truncated ellipsoidal domains of increasing size, modeling wedges of the ventricular wall. The number of subdomains (and processors) is 


\begin{tabular}{|c|c|c|c|c|c|c|c|}
\hline \multicolumn{8}{|c|}{ Bidomain solver - BJ preconditioner } \\
\hline \multirow[b]{2}{*}{ procs } & \multirow[b]{2}{*}{ dof } & \multicolumn{3}{|c|}{ non-deforming $(\mathbf{F}=\mathbf{I})$} & \multicolumn{3}{|c|}{ deforming } \\
\hline & & $\kappa_{2}$ & it & time & $\kappa_{2}$ & it & time \\
\hline 8 & 549,250 & 900.64 & 65 & 1.91 & $1.01 \mathrm{e}+3$ & 66 & 1.93 \\
\hline 27 & $1,825,346$ & $2.30 \mathrm{e}+3$ & 100 & 3.21 & $2.51 \mathrm{e}+3$ & 100 & 3.19 \\
\hline 64 & $4,293,378$ & $4.25 \mathrm{e}+3$ & 131 & 4.21 & $4.61 \mathrm{e}+3$ & 131 & 4.28 \\
\hline 125 & $8,346,562$ & $6.81 \mathrm{e}+3$ & 164 & 5.27 & $7.35 \mathrm{e}+3$ & 170 & 5.63 \\
\hline 216 & $14,378,114$ & $9.95 \mathrm{e}+3$ & 197 & 6.41 & $1.07 \mathrm{e}+4$ & 204 & 6.61 \\
\hline 343 & $22,781,250$ & $1.34 \mathrm{e}+4$ & 240 & 9.06 & $1.43 \mathrm{e}+4$ & 247 & 8.79 \\
\hline 512 & $33,949,186$ & $1.76 \mathrm{e}+4$ & 276 & 9.56 & $1.90 \mathrm{e}+4$ & 293 & 9.81 \\
\hline \multicolumn{8}{|c|}{ Bidomain solver - MHS(4) preconditioner } \\
\hline & & \multicolumn{3}{|c|}{ non-deforming $(\mathbf{F}=\mathbf{I})$} & \multicolumn{3}{|c|}{ deforming } \\
\hline procs & dof & $\kappa_{2}$ & it & time & $\kappa_{2}$ & it & time \\
\hline 8 & 549,250 & 1.11 & 3 & 1.05 & 1.11 & 3 & 1.31 \\
\hline 27 & $1,825,346$ & 1.11 & 3 & 1.19 & 1.12 & 3 & 1.17 \\
\hline 64 & $4,293,378$ & 1.12 & 3 & 1.23 & 1.13 & 3 & 1.21 \\
\hline 125 & $8,346,562$ & 1.13 & 3 & 1.31 & 1.18 & 4 & 1.49 \\
\hline 216 & $14,378,114$ & 1.18 & 4 & 1.55 & 1.20 & 4 & 1.55 \\
\hline 343 & $22,781,250$ & 1.15 & 4 & 1.62 & 1.17 & 4 & 1.66 \\
\hline 512 & $33,949,186$ & 1.14 & 4 & 1.96 & 1.17 & 4 & 1.67 \\
\hline
\end{tabular}

Table 2: Weak scaling test, Bidomain solver. Comparison of BJ and MHS(4) preconditioners on deforming and non-deforming domains. Number of processors (procs), Degrees of freedom (dof), Average condition number $\left(\kappa_{2}\right)$ per time step, average CG iteration counts (it) per time step and average CPU time in seconds (time) per time step.

increased from 8 to 512, with the largest domain being an half ellipsoid with parameters $a_{1}=b_{1}=1.5, c_{1}=4.4, a_{2}=b_{2}=2.7, c_{2}=5$, all in $\mathrm{cm}$, and $\phi_{\min }=-\pi / 2, \phi_{\max }=$ $\pi / 2, \theta_{\min }=-3 \pi / 8, \theta_{\max }=\pi / 8$. The physical dimensions of the increasing ellipsoidal domains are chosen so that the electrical mesh size $h$ is kept fixed to the value of about $h=0.01 \mathrm{~cm}$, so as to keep the local mesh on each subdomain fixed at $32 \cdot 32 \cdot 32$. The mechanical mesh size is twice the electrical one, thus on each subdomain the local mechanical mesh is $16 \cdot 16 \cdot 16$. With these choices, the global size of the discrete Bidomain system increases from about 0.5 million degrees of freedom (dof) for the smallest domain with 8 subdomains to 34 million dof for the largest domain with 512 subdomains, and the discrete non-linear elasticity system increases from about 100 thousands dof to 6.5 million. The simulation is run for 10 time steps of $0.05 \mathrm{~ms}$ during the plateau phase of the cardiac action potential, where the deformation due to the mechanical contraction is stronger.

The results regarding the mechanical solver are reported in Table 1. Both the nonlinear Newton iteration (nit) and linear GMRES iteration (lit) are completely scalable. The scalability of the GMRES iterations is achieved by the use of the AMG preconditioner. Nevertheless, the CPU times are not scalable, because they increase with the number of 


\begin{tabular}{r|r|rrrr}
\hline \multicolumn{5}{c}{ Mechanical solver - AMG preconditioner } \\
\hline procs & local dof & nit & lit & time & speedup \\
\hline 8 & 102,984 & 2 & 41 & 110.84 & - \\
16 & 51,492 & 2 & 40 & 63.61 & $1.74(2)$ \\
32 & 25,746 & 2 & 41 & 34.64 & $3.20(4)$ \\
64 & 12,873 & 2 & 39 & 23.26 & $4.76(8)$ \\
128 & 6,436 & 2 & 40 & 16.08 & $6.89(16)$ \\
256 & 3,218 & 2 & 40 & 15.50 & $7.15(32)$ \\
512 & 1,609 & 2 & 41 & 16.97 & $6.53(64)$ \\
\hline
\end{tabular}

Table 3: Strong scaling test, Mechanical solver with AMG preconditioner. Number of processors (procs), Degrees of freedom per processor (dof), average Newton iterations (nit) per time step, average GMRES iteration counts (lit) per Newton iteration and time step, average CPU time in seconds (time) per time step and standard speedup (the ideal speedup is reported in brackets).

processors, due to the setup of the AMG preconditioner at each Newton iteration.

In Table 2, we compare the BJ and MHS(4) Bidomain preconditioners, considering or neglecting ( $\mathbf{F}=\mathbf{I}$ in (7) and (11)) the influence of the mechanical tissue deformation on the conductivity tensors. The results show that both the preconditioners are robust with respect to the domain deformations due to the mechanical contraction. The BJ preconditioner is not scalable, since the condition number of the preconditioned system, the CG iterations and the CPU times increase with the number of subdomains. Instead, the MHS(4) preconditioner is completely scalable, both in terms of the mathematical quantities (condition number and CG iterations) and CPU times. In the largest simulation with 512 processors, the MHS(4) preconditioner is almost 6 times faster than the BJ preconditioner.

\subsection{Test 2: strong scaling}

In this strong scaling test, the three-dimensional cardiac domain considered is a half of truncated ellipsoid (with parameters $a_{1}=b_{1}=1.5, c_{1}=4.4, a_{2}=b_{2}=2.7, c_{2}=5$, all in $\mathrm{cm}$, and $\left.\phi_{\min }=-\pi / 4, \phi_{\max }=\pi / 4, \theta_{\min }=-\pi / 4, \theta_{\max }=\pi / 8\right)$. modeling a half the left ventricular wall, discretized by a fine electrical mesh of $128 \cdot 128 \cdot 128 Q_{1}$ finite elements, for a total amount of 2146689 nodes. The mechanical mesh size is twice the electrical one, thus the elements are $64 \cdot 64 \cdot 64$ and the nodes 274625 . Consequently, the global size of the discrete Bidomain system is fixed at about 4.2 million dof, while the size of the discrete nonlinear elasticity system is 823875 dof. The number of subdomains (= number of processors) increases from 8 to 512 . In this way, the number of dof per subdomain is reduced when the number of subdomains is increased. As in the previous test, the physical dimension of the truncated ellipsoidal domain is chosen so that the electrical mesh size $h$ is kept fixed to the value of about $h=0.01 \mathrm{~cm}$ and the simulation is run for 10 time steps of $0.05 \mathrm{~ms}$ during the plateau phase.

Table 3 reports the Newton iterations (nit), the GMRES iterations (lit), the CPU times and speedup of the mechanical solver. The speedup is defined with respect to the 8 processors 


\begin{tabular}{r|r|rrrr}
\hline \multicolumn{5}{c}{ Bidomain solver - BJ preconditioner } \\
\hline procs & local dof & $\kappa_{2}$ & it & time & speedup \\
\hline 8 & 536,672 & $3.71 \mathrm{e}+3$ & 125 & 30.50 & - \\
16 & 268,336 & $3.95 \mathrm{e}+3$ & 128 & 17.09 & $1.78(2)$ \\
32 & 134,168 & $4.02 \mathrm{e}+3$ & 129 & 8.50 & $3.59(4)$ \\
64 & 67,084 & $4.61 \mathrm{e}+3$ & 137 & 4.40 & $6.93(8)$ \\
128 & 33,542 & $5.09 \mathrm{e}+3$ & 142 & 2.21 & $13.80(16)$ \\
256 & 16,771 & $5.22 \mathrm{e}+3$ & 143 & 1.02 & $29.90(32)$ \\
512 & 8,385 & $6.29 \mathrm{e}+3$ & 154 & 0.55 & $55.45(64)$ \\
\hline \multicolumn{5}{c}{ Bidomain solver - MHS(4) preconditioner } \\
\hline procs & local dof & \multicolumn{5}{c}{$\kappa_{2}$} & it & time & speedup \\
\hline 8 & 536,672 & 1.13 & 3 & 9.18 & - \\
16 & 268,336 & 1.13 & 3 & 5.16 & $1.78(2)$ \\
32 & 134,168 & 1.14 & 3 & 2.62 & $3.50(4)$ \\
64 & 67,084 & 1.15 & 3 & 1.30 & $7.06(8)$ \\
128 & 33,542 & 1.16 & 4 & 0.72 & $12.75(16)$ \\
256 & 16,771 & 1.19 & 4 & 0.48 & $19.12(32)$ \\
512 & 8,385 & 1.20 & 4 & 0.26 & $35.31(64)$ \\
\hline
\end{tabular}

Table 4: Strong scaling test, Bidomain solver. Comparison of BJ and MHS(4) preconditioners on deforming domains. Number of processors (procs), Degrees of freedom per processor (local dof), Condition number $\left(\kappa_{2}\right)$, CG iteration counts (it), CPU time in seconds (time), Average condition number $\left(\kappa_{2}\right)$ per time step, average CG iteration counts (it) per time step, average CPU time in seconds (time) per time step and standard speedup (the ideal speedup is reported in brackets).

run as

$$
\text { speedup }(\text { procs }):=\frac{\text { time }(8)}{\text { time }(\text { procs })} \text {. }
$$

Although the Newton and GMRES iterations are completely scalable, since they remain bounded when increasing the number of processors, the speedup are quite far from the theoretical one, even with a small number of processors. Much better is the performance in terms of speedup of the Bidomain solver (see Table 4), both employing the BJ or the MHS(4) preconditioner. Thanks to small number of CG iterations, the MHS(4) preconditioner is always about 2-3 times faster than the BJ preconditioner, even if the speedup of the latter is closer to the theoretical one.

\subsection{Test 3: whole cardiac cycle}

In this last test, we study the performance of our electro-mechanical solver on a whole heartbeat simulation lasting $400 \mathrm{~ms}$. The cardiac domain is a half truncated ellipsoid as in the previous test, discretized by a fine electrical mesh of $128 \cdot 128 \cdot 64 Q_{1}$ finite elements. The mechanical mesh is much coarser and consists of $16 \cdot 16 \cdot 8$ elements. As a consequence, the Bidomain linear system dof are 2163330, while the non-linear elasticity system dof are 7803. 


\begin{tabular}{r|rr|rr|rr}
\hline \multicolumn{7}{c}{ Mechanical solver } \\
\hline prec & nit & Tnit & lit & Tlit & time & Ttime \\
\hline AMG & 3 & 549 & 52 & 29,174 & 1.02 & 203.13 \\
\hline
\end{tabular}

Table 5: Full heartbeat test, Mechanical solver with AMG preconditioner. Total number of mechanical degrees of freedom $=7,803$. Number of processors $=32$. Average Newton iterations (nit) per time step , total Newton iterations (nit), average GMRES iterations (lit) per Newton iteration and time step, total GMRES iterations (Tlit), average CPU time in seconds (time) per time step for solving one mechanical non-linear system, total CPU time in seconds (Ttime) for solving all the mechanical non-linear systems.

\begin{tabular}{r|r|rr|rr}
\hline \multicolumn{6}{|c}{ Bidomain solver } \\
\hline prec & $\kappa_{2}$ & it & Tit & time & Ttime \\
\hline BJ & $4.10 \mathrm{e}+3$ & 135 & $1,082,713$ & 5.46 & 43,707 \\
MHS(4) & 1.17 & 4 & 30,982 & 1.51 & 12,107 \\
\hline
\end{tabular}

Table 6: Full heartbeat test, Bidomain solver. Comparison of BJ and MHS(4) preconditioners on deforming domains. Total number of Bidomain degrees of freedom $=2,163,330$. Number of processors $=32$. Average Condition number $\left(\kappa_{2}\right)$ per time step, average CG iteration counts (it) per time step, total CG iteration counts (Tit), average CPU time in seconds (time) per time step for solving one Bidomain linear system, total CPU time in seconds (Ttime) for solving all Bidomain linear systems.

The number of processors is 32 . The time step size is chosen accordingly to the adaptive strategy described in [9]. The non-linear elasticity system is solved every 10 electrical time steps.

Tables 5 and 6 report the summary of the mechanical and Bidomain solver data. On a whole heart beat simulation, the results show that the MHS(4) preconditioner is about 3.6 times faster than the BJ preconditioner. Fig. 1 shows the time evolution of the condition number, CG iterations, Newton iterations, GMRES iterations and CPU times of the electromechanical solver. The plots in Panels $\mathrm{C}$ and I clearly show that the solution of the nonlinear elasticity system is harder after the excitation phase, at about $100 \mathrm{~ms}$, when the muscle contracts, and during the repolarization phase at about $300 \mathrm{~ms}$, when the muscle relaxes. Figures 2, 3 and 4 display the spatial distributions of the transmembrane and extracellular potentials on the endocardium, epicardium and on a transmural section of the deformed wedge at six different instants during the activation and plateau phases of the action potential.

\section{Conclusion}

We have constructed and studied a parallel multilevel solver for the cardiac electromechanical coupling model. The solver consists of two major steps: 1) solving the linear system deriving from the discretization of the Bidomain equations with the Conjugate Gradient (CG) method, preconditioned by Multilevel Schwarz preconditioners; 2) solving the non-linear system deriving from the discretization of the quasi-static mechanical model with 
the Newton method, where the linear Jacobian system is solved by GMRES, preconditioned by an Algebraic Multigrid preconditioner. The three-dimensional numerical tests performed on a Linux cluster up to 512 processors have shown that the mechanical non-linear solver is scalable in terms of Newton and GMRES iterations, but not in terms of CPU time. Further research is needed in order to obtain a more effective parallel non-linear solver, and a possible strategy could be to develop non-overlapping domain decomposition methods (such as BDDC or FETI-DP) for the linear Jacobian system. On the other hand, the results have shown that the Bidomain solver based on Multilevel Schwarz preconditioners is scalable both in terms of $\mathrm{CG}$ iterations and $\mathrm{CPU}$ time. Moreover, the Bidomain solver is robust with respect to the severe domain deformations induced by the mechanical contraction of the cardiac tissue.

\section{Acknowledgements}

The authors were partially supported by grants of MIUR (PRIN 201289A4LX_002) and of INdAM (Istituto Nazionale di Alta Matematica, Roma, Italy).

\section{References}

[1] D. Ambrosi, G. Arioli, F. Nobile, A. Quarteroni, Electromechanical coupling in cardiac dynamics: the active strain approach, SIAM J. Appl. Math. 71 (2011) 605-621.

[2] D. Ambrosi, S. Pezzuto, Active stress vs. active strain in mechanobiology: constitutive issues, J. Elast. 107 (2012) 199-212.

[3] B. Andreianov, M. Bendahmane, A. Quarteroni, R. Ruiz-Baier, Weak solutions and numerical approximation of a coupled system modeling cardiac electromechanics (2014). URL: http://hal . archives-ouvertes.fr/hal-00865585.

[4] S. Balay, K. Buschelman, W.D. Gropp, D. Kaushik, M. Knepley, L. Curfman McInnes, B.F. Smith, H. Zhang, PETSc users manual, Tech. Rep. ANL-95/11 - Revision 3.3, Argonne National Laboratory, 2012.

[5] D. Boffi, F. Brezzi, M. Fortin, Mixed Finite Element Methods and Applications, Springer, Berlin, 2013.

[6] S.G. Campbell, E. Howard, J. Aguado-Sierra, B.A. Coppola, J.H. Omens, L.J. Mulligan, A.D. McCulloch, R.C.P. Kerckhoffs, Effect of transmurally heterogeneous myocyte excitation-contraction coupling on canine left ventricular electromechanics, Exp. Physiol. 94 (2009) 541-552.

[7] C. Cherubini, S. Filippi, P. Nardinocchi, L. Teresi, An electromechanical model of cardiac tissue: constitutive issues and electrophysiological effects, Progr. Biophys. Molec. Biol. 97 (2008) 562-573. 


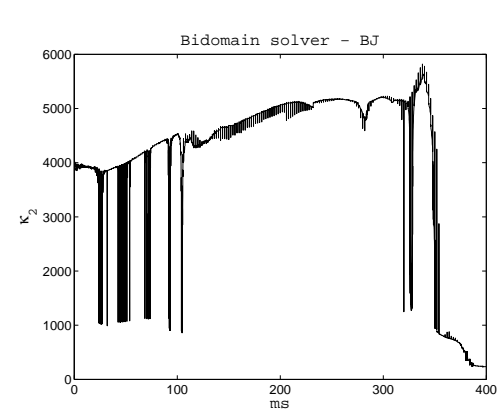

(A)

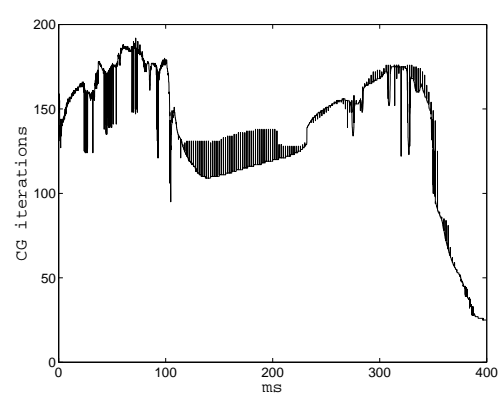

(D)

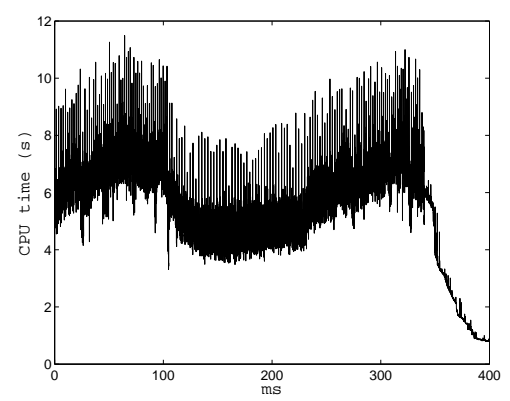

(G)

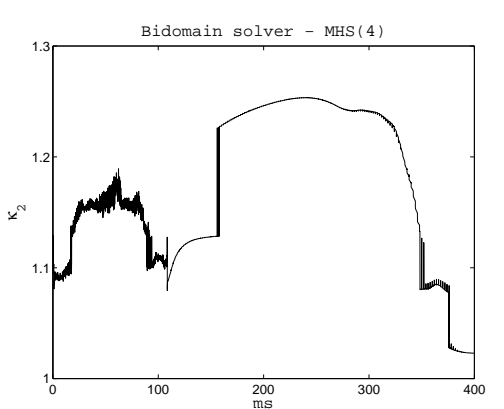

(B)

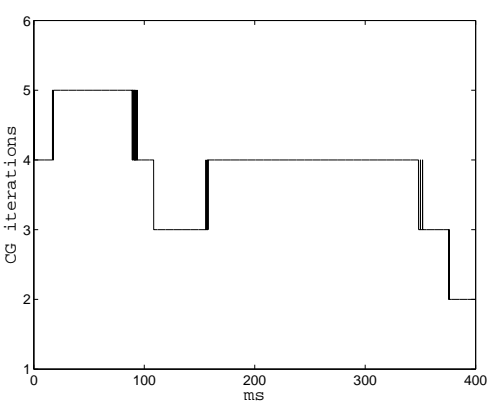

(E)

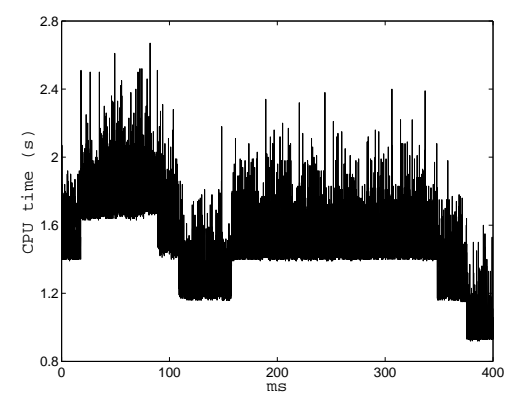

(H)

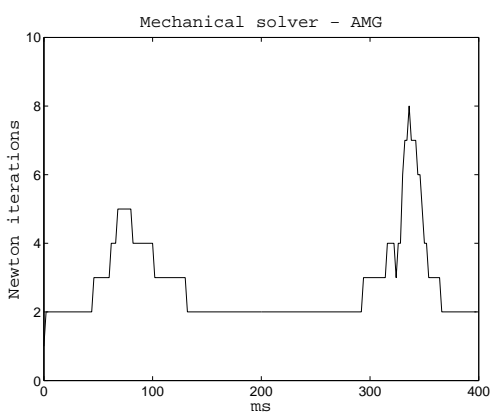

(C)

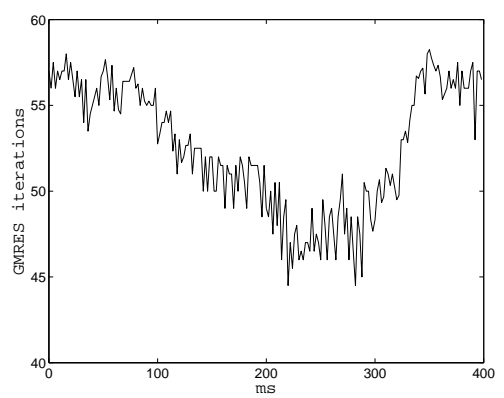

(F)

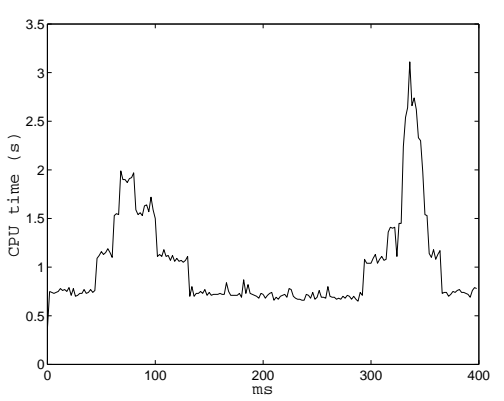

(I)

Figure 1: Full heartbeat test. Bidomain solver - BJ preconditioner: time evolution of condition number (A), CG iterations (D), CPU time in second (G) for solving one Bidomain linear system. Bidomain solver - MHS(4) preconditioner: time evolution of condition number (B), CG iterations (E), CPU time (H) for solving one Bidomain linear system. Mechanical solver - AMG preconditioner: time evolution of Newton iteration (C), GMRES iterations (F) per Newton iteration, CPU time (I) for solving one mechanical non-linear system. 

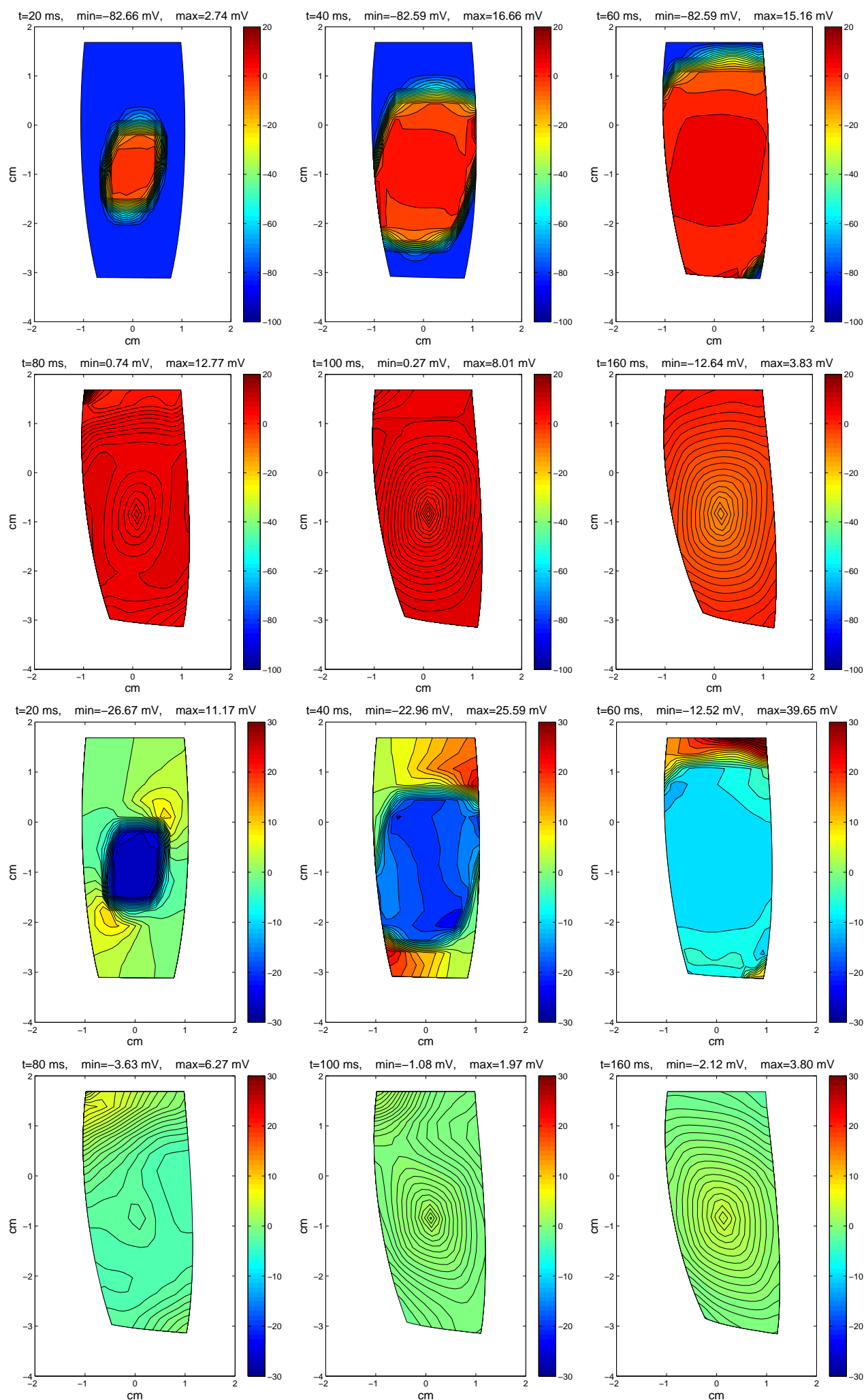

Figure 2: Full heartbeat test: Endocardial transmembrane (first and second rows) and extracellular (third and fourth rows) potential distributions on the deformed domain at different time instants. 

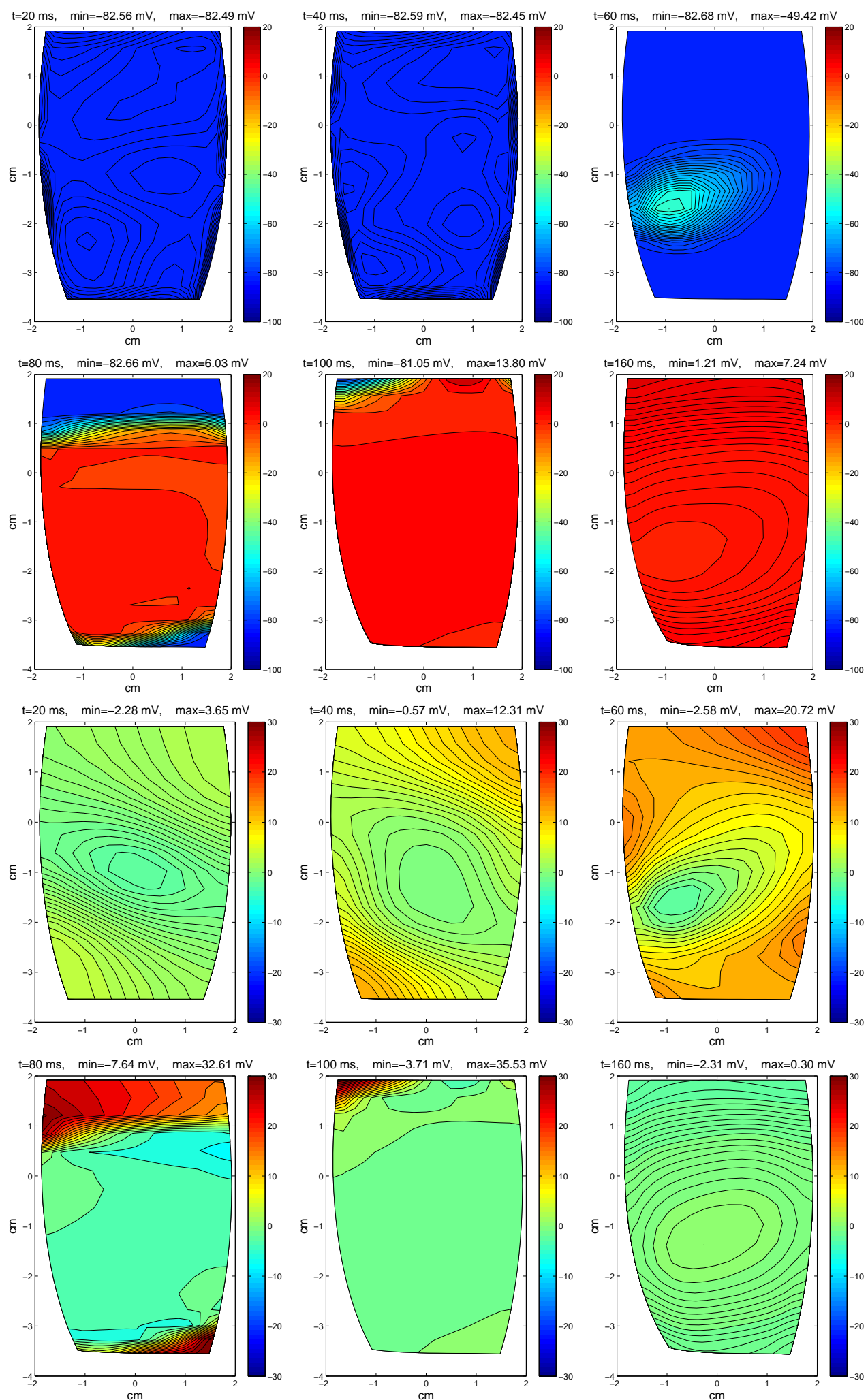

Figure 3: Full heartbeat test: Epicardial transmembrane (first and second rows) and extracellular (third and fourth rows) potential distributions on the deformed domain at different time instants. 

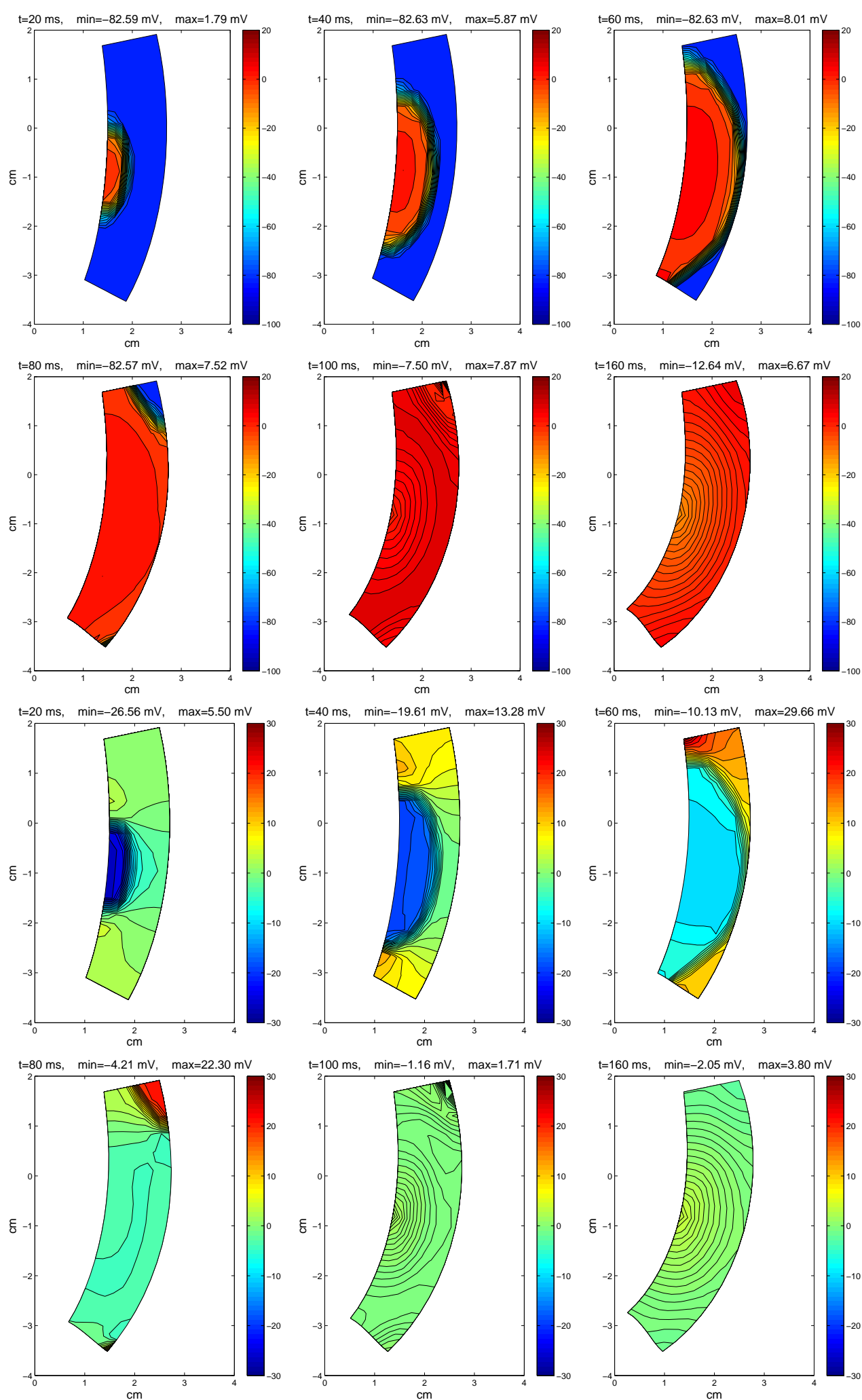

Figure 4: Full heartbeat test: Epicardial transmembrane (first and second rows) and extracellular (third and fourth rows) potential distributions on the deformed domain at different time instants. 
[8] P. Colli Franzone, L. Guerri, B. Taccardi, Modeling ventricular excitation:axial and orthotropic effects on wavefronts and potentials, Math. Biosci. 188 (2004) 191-205.

[9] P. Colli Franzone, L.F. Pavarino, A parallel solver for reaction-diffusion systems in computational electrocardiology, Math. Mod. Meth. Appl. Sci. 14 (2004) 883-911.

[10] P. Colli Franzone, L.F. Pavarino, G. Savarè, Computational Electrocardiology: mathematical and numerical modeling., in: Complex Systems in Biomedicine (A. Quarteroni et al., Eds.), Springer-Verlag, 2006, pp. 187-241.

[11] K.D. Costa, H.J. W., A.D. McCulloch, Modelling cardiac mechanical properties in three dimensions, Philos. Trans. R. Soc. London A 359 (2001) 1233-1250.

[12] H. Dal, S. Göktepe, M. Kaliske, E. Kuhl, A fully implicit finite element method for bidomain models of cardiac electromechanics, Comput. Meth. Appl. Mech. Engrg. 253 (2013) 323-336.

[13] S. Göktepe, E. Kuhl, Electromechanics of the heart - a unified approach to the strongly coupled excitation-contraction problem, Comp. Mech. 80 (2010) 227-243.

[14] L. Gerardo Giorda, L. Mirabella, F. Nobile, M. Perego, A. Veneziani, A model-based block-triangular preconditioner for the Bidomain system in electrocardiology, J. Comp. Phys. 228 (2009) 3625-3639.

[15] J.M. Guccione, K.D. Costa, A.D. McCulloch, Finite element stress analysis of left ventricular mechanics in the beating dog heart, J. Biomech. 28 (1995) 1167-1177.

[16] V.E. Henson, U.M. Yang, BoomerAMG: A parallel algebraic multigrid solver and preconditioner, Appl. Numer. Math. 41 (2002) 155-177.

[17] G.A. Holzapfel, Nonlinear Solid Mechanics, Wiley-Blackwell, Berlin, 2000.

[18] G.A. Holzapfel, R.W. Ogden, Constitutive modelling of passive myocardium. a structurally-based framework for material characterization, Phil. Trans. R. Soc. London A 367 (2009) 3445-3475.

[19] T.J.R. Hughes, The Finite Element Method, Dover Publications, Inc., New York, 2000.

[20] J.D. Humphrey, Cardiovascular Solid Mechanics, Cells, Tissues and Organs., Springer, New York, 2001.

[21] P.J. Hunter, A.D. McCulloch, H.E.D.J. ter Keurs, Modelling the mechanical properties of cardiac muscle, Progr. Biophys. Molec. Biol. 69 (1998) 289-331.

[22] P.J. Hunter, H.P. Nash, G.B. Sands, Computational electromechanics of the heart, in: Computational Biology of the Heart (A. V. Panfilov and A. V. Holden, Eds.), Wiley, 1997. 
[23] X. Jie, V. Gurev, J. Constantino, J.J. Rice, N.A. Trayanova, Distribution of electromechanical delay in the heart: insights from a three-dimensional electromechanical model, Biophys. J. 99 (2010) 745-754.

[24] X. Jie, V. Gurev, J. Constantino, J.J. Rice, N.A. Trayanova, Models of cardiac electromechanics based on individual hearts imaging data: Image-based electromechanical models of the heart, Biomech. Model Mechanobiol. 10 (2011) 295-306.

[25] X. Jie, V. Gurev, N.A. Trayanova, Mechanisms of mechanically induced spontaneous arrhythmias in acute regional ischemia, Circ. Res. 106 (2010) 185-192.

[26] R.H. Keldermann, M.P. Nash, H. Gelderblom, V.Y. Wang, A.V. Panfilov, Electromechanical wavebreak in a model of the human left ventricle, Am. J. Physiol. Heart Circ. Physiol. 299 (2010) H134-H143.

[27] R.C.P. Kerckhoffs, P.H.M. Bovendeerd, J.C.S. Kotte, F.W. Prinzen, K. Smits, T. Arts, Homogeneity of cardiac contraction despite physiological asyncrony of depolarization: a model study, Ann. Biomed. Eng. 31 (2003) 536-547.

[28] I.J. LeGrice, B.H. Smaill, L.Z. Chai, S.G. Edgar, J.B. Gavin, P.J. Hunter, Laminar structure of the heart: ventricular myocyte arrangement and connective tissue architecture in the dog, Am. J. Physiol. Heart Circ. Physiol. 269 (1995) H571-H582.

[29] C. Luo, Y. Rudy, A model of the ventricular cardiac action potential: depolarization, repolarization, and their interaction, Circ. Res. 68 (1991) 1501-1526.

[30] K.A. Mardal, B.F. Nielsen, X. Cai, A. Tveito, An order optimal solver for the discretized bidomain equations, Numer. Linear Algebra Appl. 14 (2007) 83-98.

[31] M. Munteanu, L.F. Pavarino, S. Scacchi, A scalable Newton-Krylov-Schwarz method for the bidomain reaction-diffusion system, SIAM J. Sci. Comput. 31 (2009) 3861-3883.

[32] M. Murillo, X.C. Cai, A fully implicit parallel algorithm for simulating the non-linear electrical activity of the heart, Numer. Linear Algebra Appl. 11 (2004) 261-277.

[33] M.P. Nash, P.J. Hunter, Computational mechanics of the heart. from tissue structure to ventricular function, J. Elast. 61 (2000) 113-141.

[34] M.P. Nash, A.V. Panfilov, Electromechanical model of excitable tissue to study reentrant cardiac arrhythmias, Progr. Biophys. Molec. Biol. 85 (2004) 501-522.

[35] S.A. Niederer, P.J. Hunter, N.P. Smith, A quantitative analysis of cardiac myocyte relaxation: a simulation study, Biophys. J. 90 (2006) 1697-1722.

[36] F. Nobile, A. Quarteroni, R. Ruiz-Baier, An active strain electromechanical model of cardiac tissue, Int. J. Num. Meth. Biomed. Eng. 28 (2012) 52-71. 
[37] P. Pathmanathan, M.O. Bernabeu, R. Bordas, J. Cooper, A. Garny, J.M. Pitt-Francis, J.P. Whiteley, D.J. Gavaghan, A numerical guide to the solution of the bidomain equations of cardiac electrophysiology, Progr. Biophys. Molec. Biol. 102 (2010) 136-155.

[38] P.J. Pathmanathan, J.P. Whiteley, A numerical method for cardiac mechanoelectric simulations, Ann. Biomed. Engrg. 37 (2009) 860-873.

[39] S.J. Pathmanathan, P. J. Chapman, D.J. Gavaghan, J.P. Whiteley, Cardiac electromechanics: the effect of contraction model on the mathematical problem and accuracy of the numerical scheme., Quart. J. Mech. Appl. Math. 63 (3) (2010) 375-399.

[40] L.F. Pavarino, S. Scacchi, Multilevel additive Schwarz preconditioners for the Bidomain reaction-diffusion system, SIAM J. Sci. Comput. 31 (2008) 420-443.

[41] M. Pennacchio, G. Savaré, P. Colli Franzone, Multiscale modeling for the bioelectric activity of the heart, SIAM J. Math. Anal. 37 (2006) 1333-1370.

[42] M. Pennacchio, V. Simoncini, Algebraic multigrid preconditioners for the bidomain reaction-diffusion system, Appl. Numer. Math. 59 (2009) 3033-3050.

[43] M. Pennacchio, V. Simoncini, Fast structured AMG preconditioning for the bidomain model in electrocardiology, SIAM J. Sci. Comput. 33 (2011) 721-745.

[44] G. Plank, M. Liebmann, R. Weber dos Santos, E.J. Vigmond, G. Haase, Algebraic Multigrid Preconditioner for the cardiac bidomain model, IEEE Trans. Biomed. Engrg. 54 (2007) 585-596.

[45] E.W. Remme, M.P. Nash, P.J. Hunter, Distributions of myocyte stretch, stress and work in models of normal and infarcted ventricles, in: Cardiac Mechano-Electric Feedback and Arrhythmias: From Pipette to Patient (P. Kohl, F. Sachse and M. R. Franz, Eds.), UK: Saunders-Elsevier, 2005, pp. 389-391.

[46] J.J. Rice, P.P. de Tombe, Approaches to modeling crossbridges and calcium-dependent activation in cardiac muscle, Progr. Biophys. Molec. Biol. 85 (2004) 179-195.

[47] J.J. Rice, F. Wang, D.M. Bers, P.P. de Tombe, Approximate model of cooperative activation and cross bridge cycling in cardiac muscle using ordinary differential equations, Biophys. J. 95 (2008) 2368-2390.

[48] S. Rossi, R. Ruiz-Baier, L.F. Pavarino, A. Quarteroni, Orthotropic active strain models for the numerical simulation of cardiac biomechanics, Int. J. Num. Meth. Biomed. Engrg. 28 (2012) 761-788.

[49] J. Sainte-Marie, D. Chapelle, C. R., M. Sorine, Modeling and estimation of cardiac electromechanical activity, Comp. Struct. 84 (2006) 1743-1759. 
[50] S. Scacchi, A hybrid multilevel Schwarz method for the bidomain model, Comput. Meth. Appl. Mech. Engrg. 197 (2008) 4051-4061.

[51] S. Scacchi, A multilevel hybrid Newton-Krylov-Schwarz method for the Bidomain model of electrocardiology, Comput. Meth. Appl. Mech. Engrg. 200 (2011) 717-725.

[52] H. Schmid, M.P. Nash, A.A. Young, P.J. Hunter, Myocardial material parameter estimation-A comparative study of simple shear, Trans. ASME 128 (2006) 742-750.

[53] B.F. Smith, P. Bjørstad, W.D. Gropp, Domain Decomposition: Parallel Multilevel Methods for Elliptic Partial Differential Equations, Cambridge University Press, 1996.

[54] J. Sundnes, G.T. Lines, K.A. Mardal, A. Tveito, Multigrid block preconditioning for a coupled system of partial differential equations modeling the electrical activity in the heart, Comput. Meth. Biomech. Biomed. Engrg. 5 (2002) 397-409.

[55] J. Sundnes, S. Wall, H. Osnes, T. Thorvaldsen, A.D. McCulloch, Improved discretisation and linearisation of active tension in strongly coupled cardiac electro-mechanics simulations, Comput. Meth. Biomech. Biomed. Engrg. (2012). doi:10. 1080/10255842. 2012.704368.

[56] A. Toselli, O.B. Widlund, Domain Decomposition Methods: Algorithms and Theory, Springer-Verlag, Berlin, 2004.

[57] T.P. Usyk, R. Mazhari, A.D. McCulloch, Effect of laminar orthotropic myofiber architecture on regional stress and strain in the canine left ventricle, J. Elast. 61 (2000) $143-164$.

[58] M. Vazquez, R. Aris, G. Houzeaux, R. Aubry, P. Villar, J. Garcia-Barnes, D. Gil, F. Carreras, A massively parallel computational electrophysiology model of the heart, Int. J. Numer. Meth. Biomed. Engrg. 27 (2011) 1911-1929.

[59] F.J. Vetter, A.D. McCulloch, Three-dimensional stress and strain in passive rabbit left ventricle: a model study, Ann. Biomed. Engrg. 28 (2000) 781-792.

[60] E.J. Vigmond, R. Weber dos Santos, A.J. Prassl, M. Deo, G. Plank, Solvers for the cardiac bidomain equations, Progr. Biophys. Molec. Biol. 96 (2008) 3-18.

[61] J.P. Whiteley, M.J. Bishop, D.J. Gavaghan, Soft tissue modelling of cardiac fibres for use in coupled mechano-electric simulations, Bull. Math. Biol. 69 (2007) 2199-2225.

[62] S. Zampini, Balancing Neumann-Neumann methods for the cardiac Bidomain model, Numer. Math. 123 (2013) 363-393.

[63] S. Zampini, Dual-primal methods for the cardiac bidomain model, Math. Mod. Meth. Appl. Sci. 24 (2014) 667-696. 